\title{
Evaluation of bone marrow in patients with pancytopenia
}

\author{
Pathak $\mathrm{R}^{1}$, Jha $\mathrm{A}^{1}$, Sayami $\mathrm{G}^{1}$

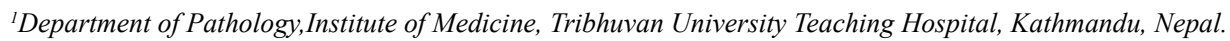

\author{
Keywords: \\ Bone marrow; \\ Pancytopenia; \\ Hypoplastic; \\ Hematological \\ malignancy; \\ Megaloblastic anemia
}

\begin{abstract}
Background: Pancytopenia is a common hematological finding resulting from varieties of disease processes that require evaluation of bone marrow. This study was carried out to evaluate bone marrow findings in patients presenting with pancytopenia.
\end{abstract}

Materials and Method: This was a prospective cross sectional study carried out to identify the causes of pancytopenia based on bone marrow examination. Bone marrow examinations were performed in 503 cases for different indications over a period of one year.

Results: One hundred and two $(20.27 \%)$ cases fulfilled the criteria of pancytopenia. Trephine biopsy was possible only in 48 cases. In $75 \%$ cases aspiration findings were similar to biopsy. Mean age of patients was 38.8 years. Maximum number of cases was seen in age group of 15-30 years. Hypoplastic anemia was the commonest cause followed by hematological malignancies, megaloblastic anemia, leishmaniasis and Gaucher disease. Bone marrow examination alone was able to establish the diagnosis in $76.5 \%$ cases. In rest marrow findings were nonspecific and in $4.9 \%$ cases findings were normal.

Conclusion: Bone marrow aspiration coupled with trephine biopsy can diagnose majority but not all the cases of pancytopenia. Hypoplastic anemia, hematological malignancies and megaloblastic anemia are the commonest causes of pancytopenia. Maximum diagnostic yield can be achieved by correlation with clinical findings, peripheral blood findings and with other laboratory and radiological parameters.

\section{INTRODUCTION}

Pancytopenia is reduction in all three major formed elements of the blood that may result from a number of disease processes. Pancytopenia develops by varieties of mechanisms such as decrease in hematopoietic cell production as a result of destruction of marrow by toxins or suppression of normal marrow growth and differentiation. ${ }^{1}$ Other mechanisms include ineffective hematopoiesis with cell death in the marrow, formation of defective cells that are rapidly removed from the circulation, sequestration

\section{Correspondence:}

Dr Abhimanyu Jha, MD

Associate Professor, Department of Pathology, Institute of Medicine, Tribhuvan University Teaching Hospital, Kathmandu, Nepal.

Email:jhaabhimanyu@yahoo.com or destruction of cells by the action of antibodies and trapping of normal cells in hypertrophied and overactive reticuloendothelial system. ${ }^{1}$ Marrow cellularity and composition differ in relationship to the cause. The marrow is generally hypocellular in cases of pancytopenia caused by a primary production defects. Cytopenias resulting from ineffective hematopoiesis, increased peripheral utilization or destruction of cells and bone marrow invasive processes are usually associated with a normocellular or hypercellular marrow. ${ }^{2}$

The presenting symptoms are usually attributable to anemia or thrombocytopenia. Leukopenia is uncommon at initial presentations of the patient, but can become the most serious threat to life during the subsequent course of 
the disorders. Common clinical manifestations are pallor, fatigue, splenomegaly, lymphadenopathy, fever, bleeding, weight loss, hepatomegaly and jaundice. ${ }^{3}$

Marrow aspirate has been primarily utilized for cytological assessment. Trephine biopsy; on the other hand, allow for studies of the marrow's overall cellularity, detection of focal lesions, and extent of infiltration by various pathologic entities. ${ }^{4}$ Rationale for this study is to identify the diagnostic reliability of bone marrow aspiration and biopsy in diagnosing various causes of pancytopenia.

\section{MATERIALS AND METHODS}

This was a cross-sectional comparative prospective study conducted over a period of one year (January 2009 to December 2009) in the department of Pathology, Tribhuvan University Teaching hospital, Kathmandu, Nepal. All the cases of pancytopenia with hemoglobin less than 10 $\mathrm{gm} / \mathrm{dl}$, total leucocyte count of less than $4000 / \mathrm{mm}^{3}$ and platelet count less than $150,000 / \mathrm{mm}^{3}$ were included in the study. Cases of chemotherapy induced pancytopenia were excluded. Relevant clinical findings of the patients were obtained. All the patients fulfilling the criteria were subjected to complete blood count, and peripheral blood smear examination. After taking informed consent bone marrow aspiration (BMA) was performed from posterior iliac crest of the patients. However, trephine biopsy was performed only in 48 cases. BMA smears were stained with Wright stain for microscopy and when required special stains such as periodic acid-Schiff and myeloperoxidase stain were performed. Trephine biopsy specimens were fixed in Bouin's fixative and hematoxylin and eosin stained sections were examined. Data were analyzed using Microsoft Excel.

\section{RESULTS}

During the study period of one year 503 bone marrow examinations (BME) were performed for various indications. One hundred and two cases of pancytopenia fulfilling the inclusion criteria were included in the study. Five cases of pancytopenia associated with chemotherapy were excluded from the study. Bone marrow trephine biopsy was possible only in 48 cases of pancytopenia. 50 cases were male and 52 cases were female. Maximum number of cases was seen in age group of $15-30$ years $(29.4 \%)$ followed by $30-45$ years $(28.4 \%)$.

Table 1: Bone marrow aspiration findings in 102 cases of pancytopenia

\begin{tabular}{lcc}
\hline \multicolumn{1}{c}{ Diagnosis } & $\begin{array}{c}\text { No. of } \\
\text { cases }\end{array}$ & $\begin{array}{c}\text { Percentage } \\
(\%)\end{array}$ \\
\hline Hypoplastic marrow & 33 & 32.3 \\
Erythroid hyperplasia & 21 & 20 \\
\hline Megaloblastic anemia & 12 & 11.7 \\
Normocellular marrow & 6 & 5.8 \\
\hline Myelodysplastic syndrome (MDS) & 6 & 5.8 \\
Acute lymphoblastic leukemia (ALL) & 5 & 4.9 \\
Acute myeloblastic leukemia (AML) & 4 & 3.9 \\
Non-Hodgkin lymphoma (NHL) & 2 & 1.9 \\
\hline Leishmaniasis & 2 & 1.9 \\
\hline Plasmacytosis & 2 & 1.9 \\
Gaucher disease & 1 & 0.9 \\
Reactive myeloid hyperplasia & 1 & 0.9 \\
Eosinophilia & 1 & 0.9 \\
Inconclusive & 6 & 5.8 \\
\hline Total & $\mathbf{1 0 2}$ & $\mathbf{1 0 0}$ \\
\hline
\end{tabular}

Table 2: Bone marrow biopsy findings

\begin{tabular}{|c|c|c|c|c|c|}
\hline \multirow{2}{*}{ Diagnosis } & \multirow{2}{*}{$\begin{array}{l}\text { No. of } \\
\text { cases }\end{array}$} & \multirow{2}{*}{ Finding same as BMA } & \multicolumn{3}{|c|}{ Finding different to BMA } \\
\hline & & & HA & NHL & MDS \\
\hline Hypoplastic anemia (HA) & 13 & 13 & & & \\
\hline Erythroid hyperplasia & 08 & 04 & 03 & 01 & \\
\hline Megaloblastic anemia & 05 & 05 & & & \\
\hline Normal marrow & 04 & 03 & 01 & & \\
\hline MDS & 03 & 03 & & & \\
\hline ALL & 02 & 02 & & & \\
\hline AML & 02 & 02 & & & \\
\hline NHL & 01 & 01 & & & \\
\hline Leishmaniasis & 01 & 01 & & & \\
\hline Plasmacytosis & 01 & 00 & 01 & & \\
\hline Gaucher disease & 01 & 01 & & & \\
\hline Reactive myeloid hyperplasia & 01 & 01 & & & \\
\hline Eosinophilia & 00 & 00 & & & \\
\hline Inconclusive & 06 & 00 & 05 & & 01 \\
\hline Total & 48 & 36 & 10 & 1 & 1 \\
\hline
\end{tabular}


Hypoplastic marrow was the commonest aspiration finding followed by erythroid hyperplasia, hematological malignancies and megaloblastic anemia (fig.1). Leishmaniasis (fig.2), plasmacytosis, Gaucher disease, eosinophilia and reactive myeloid hyperplasia were other aspiration findings (Table 1).

Table 3: Causes of pancytopenia based on bone marrow aspiration and biopsy findings

\begin{tabular}{llcc}
\hline S.N. & \multicolumn{1}{c}{ Diagnosis } & $\begin{array}{c}\text { Total No. of } \\
\text { cases }\end{array}$ & $\begin{array}{c}\text { \% of total } \\
\text { cases }\end{array}$ \\
\hline 1 & Hypoplastic anemia & 43 & 42.1 \\
2 & Megaloblastic anemia & 12 & 11.7 \\
3 & Acute leukemia & 09 & 8.8 \\
\hline 4 & MDS & 07 & 6.8 \\
\hline 5 & NHL & 03 & 2.9 \\
\hline 6 & Leishmaniasis & 02 & 1.9 \\
\hline 7 & Gaucher disease & 01 & 0.9 \\
\hline 8 & Plasma cell myeloma & 01 & 0.9 \\
\hline 9 & Undiagnosed & 24 & 23.52 \\
\hline & Total & $\mathbf{1 0 2}$ & $\mathbf{1 0 0}$ \\
\hline
\end{tabular}

Out of 48 cases of available bone marrow biopsies (BMB) 36 cases had same findings as it was seen in aspiration. In 12 cases biopsy findings were different from the BMA findings (Table 1-2). Additional 10 cases of hypoplastic anemia was diagnosed in $\mathrm{BMB}$, the findings in these cases in BMA were inconclusive in 5 cases, erythroid hyperplasia $(\mathrm{EH})$ in 3 cases, plasmacytosis and normal marrow in 1 case each. Non-Hodgkin lymphoma (NHL; fig.3) and myelodysplastic syndrome (MDS; fig.4) 1 case each were diagnosed following BMB which the aspiration did not reveal (Table 2).

Twenty four $(23.52 \%)$ cases of pancytopenia remained undiagnosed in this study. Commonest cause of pancytopenia was hypoplastic anemia followed by hematological malignancies, and megaloblastic anemia. Two cases of leishmaniasis and 1 case Gaucher disease were also detected (Table 3). One case of plasma cell myeloma was also diagnosed based on BMA findings and clinicoradiological findings. Bone marrow aspiration revealed only $13 \%$ plasma cells, however, there was hypercalcemia and X-Ray skull revealed lytic lesions.

Table 4: Comparison of causes of pancytopenia in children and adults

\begin{tabular}{|c|c|c|c|}
\hline Bone marrow findings & Children ( $\leq 15$ yrs) No. of cases & Adult ( $>15$ yrs) No. of cases & Total \\
\hline Hypoplastic anemia (HA) & 3 & 40 & 43 \\
\hline Megaloblastic anemia & 1 & 11 & 12 \\
\hline MDS & 0 & 7 & 7 \\
\hline ALL & 2 & 3 & 5 \\
\hline AML & 0 & 4 & 4 \\
\hline NHL & 0 & 3 & 3 \\
\hline Leishmaniasis & 0 & 2 & 2 \\
\hline Plasmacytosis & 0 & 1 & 1 \\
\hline Gaucher disease & 0 & 1 & 1 \\
\hline Total & 6 & 72 & 78 \\
\hline
\end{tabular}

Table 5: Common causes of pancytopenia in different studies

\begin{tabular}{|c|c|c|c|c|c|}
\hline Study & Country & Year & No. of cases & Commonest cause $(\%)$ & Second most common cause $(\%)$ \\
\hline Jha et $\mathrm{al}^{5}$ & Nepal & 2007 & 148 & Hypoplastic anemia (29.05) & Megaloblastic anemia (23.64) \\
\hline Makaju et $\mathrm{al}^{8}$ & Nepal & 2009 & 28 & $\begin{array}{l}\text { Megaloblastic anemia } \\
(42.85)\end{array}$ & Aplastic anemia (35.71) \\
\hline $\begin{array}{l}\text { International } \\
\text { agranulocytosis and aplastic } \\
\text { anemia study group }\end{array}$ & Israel and Europe & 1987 & 319 & Hypoplastic anemia (52.7) & $\operatorname{MDS}(4.5)$ \\
\hline Keisu and ost ${ }^{10}$ & Israel and Europe & 1990 & 100 & $\begin{array}{l}\text { Neoplastic disease, } \\
\text { radiation (32) }\end{array}$ & Hypoplastic anemia (19) \\
\hline Hossain et $\mathrm{al}^{11}$ & Bangladesh & 1992 & 50 & Hypoplastic anemia & Chronic malaria and kalazar \\
\hline Varma and Dash ${ }^{12}$ & India & 1992 & 202 & Hypoplastic anemia (40.6) & Megaloblastic anemia (23.3) \\
\hline Tilak and Jain ${ }^{13}$ & India & 1999 & 77 & Megaloblastic anemia (68) & Hypoplastic anemia (7.7) \\
\hline Kumar et $\mathrm{al}^{14}$ & India & 1999 & 166 & Hypoplastic anemia (29.51) & Megaloblastic anemia (22.3) \\
\hline Khodke et al ${ }^{15}$ & India & 2000 & 50 & Megaloblastic anemia (44) & Hypoplastic anemia (14) \\
\hline Present study & Nepal & 2010 & 102 & Hypoplastic anemia (42.15) & Hematological malignancies (19.4) \\
\hline
\end{tabular}




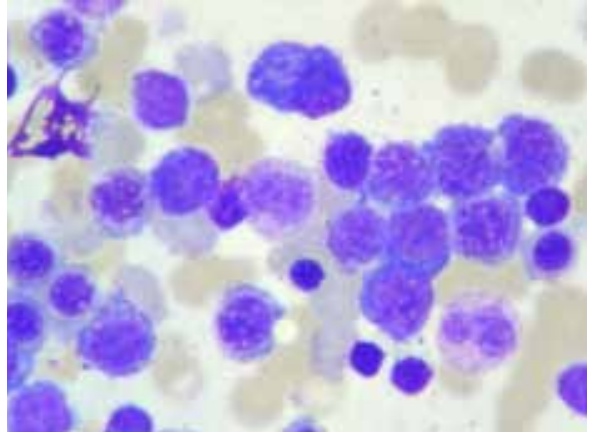

Figure 1: Megaloblastic maturation of erythroid precursors (Wright stain, X1000).

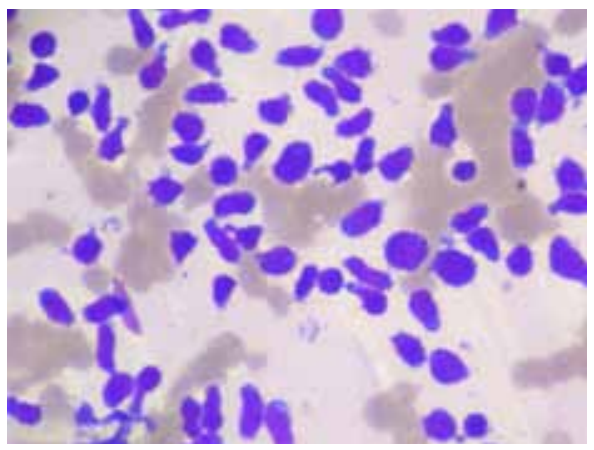

Figure 3: Non Hodgkin lymphoma. Mostly small cleaved lymphocytes (Wright stain, X400).

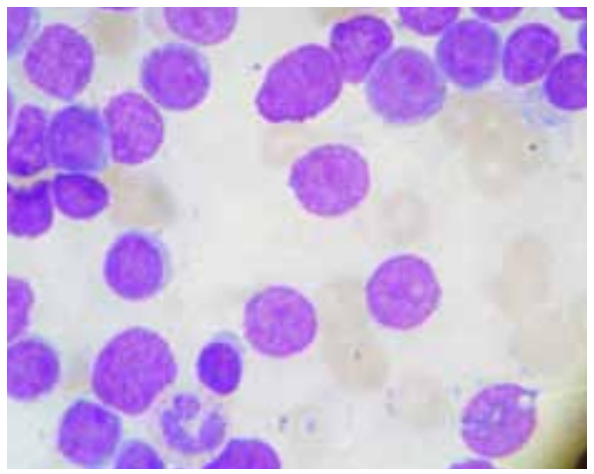

Figure 5: Acute lymphoblastic leukemia. Mostly large lymphoblasts with high nuclear cytoplasmic ratio (Wright stain, X1000).

Among hematological malignancies $(18.62 \%, 19$ cases $)$ acute leukemia was the commonest followed by MDS and NHL (Table 3,4 and fig.1). Acute lymphoblastic leukemia (ALL; fig.5) exceeded acute myeloid leukemia (AML; fig.6) in number and both were more common among adults (Table 4). MDS constituted 2 cases each of refractory anemia with excess blast-1 (RAEB-1), refractory anemia with excess of blast-2 (RAEB-2) and refractory cytopenia with multilineage dysplasia (RCMD) and 1 case of MDS was associated with marrow fibrosis, however, WHO typing was not possible. A comparison of causes of pancytopenia in children and adult is shown in table 4.

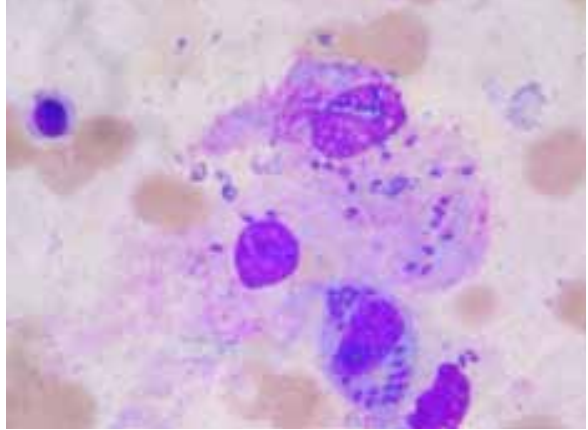

Figure 2: Leishmaniasis. Intracellular and extracellular $L D$ bodies (Wright stain, X1000).

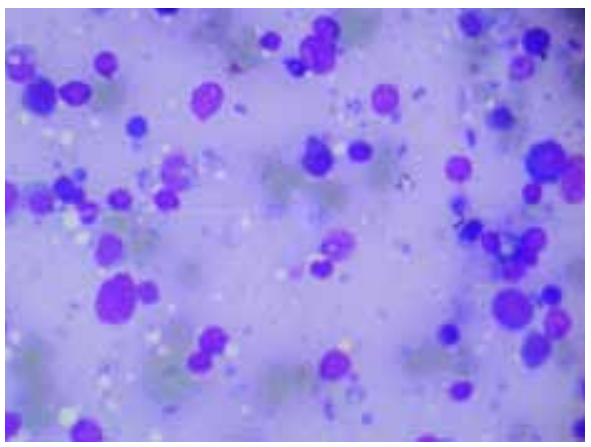

Figure 4: Myelodysplastic syndrome. Scattered blasts in a background of dyspoietic erythroid \& myeloid precursors and giant thombocytes (Wright stain, X400).

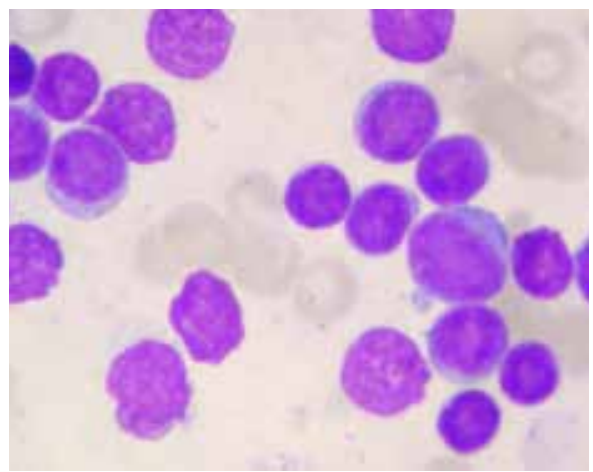

Figure 6: Acute myeloblastic leukemia. Myeloblasts with fine cytoplasmic granules (Wright stain, X1000).

\section{DISCUSSION}

Pancytopenia is a common hematological finding with variable clinical presentations. It often poses diagnostic challenge to physician and the knowledge of accurate etiologies of this condition is crucial in the management of the patient.

In this study out of 503 cases of BME, $21.27 \%$ cases were indicated for pancytopenia. In a study conducted by Jha et al and Bashawri et al the frequency of BME for evaluation of pancytopenia was $17.34 \%$ and $11.9 \%$ respectively. ${ }^{5,6}$ 
Female patients slightly outnumbered the male (1:1.04) and this was similar to study of Aziz et al. ${ }^{7}$ However, in the study of Makaju et al and Jha et al M:F were 1.5:1 and 1.3:1 respectively. ${ }^{5}$

Most cases were seen in adults (peak age 15-30 years) and only 8 cases were seen in children. BME revealed the cause of pancytopenia in 78 cases; out of that 6 were children (Table 4); which is very low as compared to the study by Jha et al, where out of 148 cases; 42 cases were children. ${ }^{5}$

In this study, most common cause of pancytopenia was hypoplastic anemia followed by hematological malignancies, megaloblastic anemia, leishmaniasis and Gaucher disease. BME was able to diagnose the cause of pancytopenia in $76.47 \%$ of the cases which was similar to study of Jha et al. ${ }^{5}$ Twenty three percent of cases in this study remained undiagnosed and BMA findings in those cases were erythroid hyperplasia, eosinophilia and reactive myeloid hyperplasia, and in 5 cases marrow finding was normal. Table 5 compares common causes of pancytopenia in different studies. Hypoplastic anemia was commonest cause of pancytopenia, ${ }^{5,8-12}$ however, in some studies hypoplastic anemia was next to megaloblastic anemia and latter was the commonest cause of pancytopenia. ${ }^{11}$ But in a study by Keisu et al neoplastic disease was the commonest cause of pancytopenia, unlike present study in which it was second in the list. ${ }^{9}$ The high frequency of malaria and kalazar in their study may be due to study done in an endemic area. In our study only 2 cases of pancytopenia showed leishmaniasis; and malaria was not detected. Only a single study showed MDS as the second commonest cause of pancytopenia. ${ }^{8}$

In present study 33 cases of HA was diagnosed in BMA, and when correlated with BMB it increased to 43 . Of these 33 cases of hypoplastic anemia diagnosed in BMA, 13 were confirmed by biopsy and in 20 cases diagnosis was based on BMA alone. Another 10 cases of HA was diagnosed by $\mathrm{BMB}$, in these cases BMA findings were different.

In 12 cases, $\mathrm{BMB}$ findings were different from the aspiration findings. In these 12 cases BMA findings were erythroid hyperplasia, normal marrow, and plasmacytosis; and 5 cases were inconclusive in aspiration. BMB and BMA are complementary to each other and it is well proven and stands true in present study as well. A diagnosis of total 12 cases was contributed by BMB and in these cases BMA findings were nonspecific or inconclusive (Table 2). This study provides a very important message that in all the cases of pancytopenia both bone marrow aspiration and biopsy must be performed.

Twelve cases of pancytopenia revealed megaloblastic anemia in BMA which was supported by BMB in five cases. This constituted $11.76 \%$ of total cases of pancytopenia. Megaloblastic anemia is a common cause of pancytopenia in various other studies. ${ }^{11}$ The high prevalence of nutritional anemia has been cited for the increased frequency of megaloblastic anemia. ${ }^{7}$ Most cases was seen in adult except one case. The casue of megaloblastic anemia was not studied in this study and evaluation of serum folate or vitamin B12 was not available in this study.

HMg were seen in $18.5 \%$ (19 cases) cases of pancytopenia (Table 4) and constituted second most common diagnosis of pancytopenia following hypoplastic anemia. Seventeen cases were diagnosed in BMA and in 8 cases BMB was available to support the diagnosis. Additional 2 cases was contributed by $\mathrm{BMB}$, in these 2 cases aspiration findings was erythroid hyperplasia in one and another case was inconclusive in aspiration. Out of 19 cases of hematological malignancies 9 cases were acute leukemia and was commonest $\mathrm{HM}$ causing pancytopenia. Acute leukemia constituted $8.8 \%$ of total cases of pancytopenia in present study which is very low as compared to study of Jha et al in which it constituted $19.59 \%$ of total cases of pancytopenia. ${ }^{5}$ However, in study of Kumar et al no cases of acute leukemia detected $^{12}$ and in study of Tilak et al only 1 case of acute leukemia was detected as a cause pancytopenia. ${ }^{11}$ On the other hand in the study of Bashwari et al the main indication of BME in case of pancytopenia was investigation of acute leukemia. ${ }^{6}$ Acute leukemia constituted third most common cause of pancytopenia in the study of Savage et $\mathrm{al}^{13}$ and similar finding was seen in study of Varma and Dash. ${ }^{10}$ In the study of Aziz et al acute leukemia constituted almost $10 \%$ of cases of pancytopenia and was third most common cause of pancytopenia. ${ }^{7}$

ALL and AML constituted 5 and 4 cases respectively. Only 2 cases of ALL were seen in children rest of the acute leukemia was seen in adult. In study of Savage et al, acute leukemia with pancytopenia was seen mostly in children unlike present study. ${ }^{13}$ Following AML next in frequency were MDS and NHL. Majority of hematological malignancies were seen in adults except for 2 cases of ALL.

A total 6 cases of MDS were diagnosed from aspiration and one on biopsy which were inconclusive on BMA. Though MDS is second most common cause of pancytopenia in studies by International agranulocytosis and aplastic anemia group but the frequency was $4.5 \%$ which is nearly similar to our study $(6.8 \%){ }^{8}$ Age range was $38-75$ yrs. Though MDS is common in older individuals, less than $10 \%$ of case occurs before the age of $50 .{ }^{14}$ In present study none of the MDS cases were seen children. BMB must be done in conjunction with aspiration in suspected MDS cases, as it was evident by 1 case in this study where aspiration was inconclusive and diagnosis was confirmed in biopsy.

Three cases of NHL were diagnosed as a cause of pancytopenia. Two of the cases were diagnosed in aspiration and in one case biopsy was available which supported the diagnosis. In one case aspiration showed erythroid 
hyperplasia, however, biopsy unmasked the diagnosis.

Two cases of leishmaniasis were diagnosed in BMA. Both of them were from the Kathmandu valley. Whether they visited in endemic area recently was not known as leishmaniasis is not prevalent in the Kathmandu valley. Kalazar is one of the cause of pancytopenia and frequency is very high in some studies done in india and Pakistan. ${ }^{15}$ Visceral leishmaniasis is endemic in some parts of india and may simulate and cause many hematological disorders like pancytopenia, myelofibrosis, MDS and hemophagocytosis as mentioned by Sud et al. ${ }^{16}$

In this study one case of Gaucher disease was diagnosed in a 20 yrs female. Study of Ahmad et al showed that storage disorders were diagnosed in $2.4 \%$ cases of pancytopenia. Out of 590 bone marrow aspiration done, there were 10 cases of Niemann Pick disease (1.6\%) and 5 cases of Gaucher disease (0.8\%).20 Gaucher disease was seen in $0.7 \%$ of cases of patients with pancytopenia in the study of Ikram et al. ${ }^{18}$

One case was diagnosed as plasma cell myeloma in this study which was similar to study by Jha et al. ${ }^{5}$ Patient was a male of 52 years. BMB was not available in this case, however, other clinical findings (lytic bony lesions, hypercalcemia, anemia) were present and increased $M$ protein was seen in urine. In another case of plasmacytosis, aspiration showed possibility of plasma cell myeloma but other clinical features and laboratory findings were not suggestive for this disease and biopsy in this case showed hypoplastic marrow. In case of suspected plasma cell myeloma BMB is strongly recommended. ${ }^{19}$ However in a study of Charles et al, who compared 79 simultaneous marrow aspirates and trephine biopsies from multiple myeloma patients, suggested that particulate aspirates may be at least as sensitive as trephine biopsies for detecting myeloma. ${ }^{20}$

Out of 102 cases of pancytopenia, 20\% of BMA showed erythroid hyperplasia, however, after correlation with biopsy, it reduced to $16.6 \%$. Erythroid hyperplasia was normoblastic in present study too. Erythroid hyperplasia by itself is not the cause of pancytopenia. Relationship of erythroid hyperplasia to pancytopenia is uncertain. Some of these cases may represent one phase in the evolution of hypoplasia, while some may be case of refractory anemia. Hypercellular or normocellular marrow in cases of pancytopenia can also be seen in cases with ineffective hematopoiesis with cell death within the marrow. ${ }^{1}$ Focal hyperplasia of erythroid or granulocytic cells at a similar stage of maturation may be observed in hot spot. ${ }^{19,21}$ A correlation with BME, clinical parameters and other laboratory parameters are required to trace the cause of pancytopenia in these cases. A possible hypersplenism needs to be ruled out in addition to different haemolytic anemias in cases of marrow showing erythroid hyperplasia. Splenomegaly was seen in five cases of erythroid hyperplasia in present study.

\section{CONCLUSION}

BMA coupled with BM biopsy can diagnose majority but not all the cases of pancytopenia. Major differential diagnostic considerations of pancytopenia are hypoplastic anemia, hematological malignancies and megaloblastic anemia. PBS and correlation with other laboratory and radiological parameters are required to arrive at diagnosis.

\section{REFERENCES}

1. Williams MD. Pancytopenia, aplastic anemia and pure red cell aplasia. In: Lee RG, Foerster J, Lukens J, Paraskevas F, Greer JP, Rodgers GM, (eds). Wintrobe's Clinical Haematology 10th edn. Williams and Wilkins;1997. pp1449-76.

2. Mckenna RW, Kroft SH. Disorders of bone marrow. Mills SE, Carter D, Greenson JK, Reuters VE, Stoler MH, (eds). In: Sternberg's Diagnostic Surgical pathology. 5th edn. Kluwer Lippincott; Wiliams and Wilkins; 2010. pp611-4.

3. Tariq M, Khan N, Basri R, Amin S. Aetiology of pancytopenia. Professional Med J 2010;17:252-6.

4. Trewhitt KG. Bone marrow aspiration and biopsy: Collection and interpretation. Oncol Nurs Forum 2001;28:1409-15.

5. Jha A, Sayami G, Adhikari RC, Panta AD, Jha R. Bone Marrow Examinations in cases of Pancytopenia. J Nepal Med Assoc 2008;47:12-7.

6. Bashwri LA. Bone marrow examination. Indications and diagnostic value. Saudi Medi J 2002;23:191-6.

7. Aziz T, Liaquat Ali L, Ansari T, Liaquat HB, Shah S, Jamal AJ. Pancytopenia: Megaloblastic anemia is still the commonest cause. Pak J Med Sci 2010;26:1132-6.

8. Incidence of aplastic anemia, the relevance of diagnostic criteria. International agranulocytosis and aplastic anemia study group. Blood 1987;70:1718-21.

9. Keisu M, Ost A. Diagnosis in patients with severe pancytopenia suspected of having aplastic anemia. Eur J Haematol 1990;45:11-4.

10. Varma N, Dash S. A reappraisal of underlying pathology in adult patients presenting with pancytopenia. Trop Geogr Med 1992;44:3227.

11. Tilak N, Dash S. Reappraisal of underlying pathology in adult patients presenting with pancytopenia. Trop Georg Med 1999;44:322-7.

12. Kumar R, Kalra SP, Kumar H, Anand AC, Madan H. Pancytopenia A six year study. J Asoc Physicians India 2001;49:1078-81.

13. Savage DG, Allen RH, Gangaidzo IT, Levy LM, Gwanzura C. Pancytopenia in Zimbabwe. Am J Med Sci 1999;317:22-32.

14. Brunning RD, Orazi A, Germing U, Le Beau MM, Porwit A, Baumann I. Myelodysplastic syndromes / neoplasms: overview. In: Swerdlow SH, Campo E, Harris LN, Jaffe SE, Pileri SA, Stein H editors, (eds). World health organization classification of tumors of hematopoietic and lymphoid tumors. Lyon: International agency for research on cancer; 2008.pp88-93.

15. Dhingra KK, Gupta P, Saroha V, Setia N, Khurana NN, Singh T. Morphological findings in bone marrow biopsy and aspirate smears of visceral kala azar: A review. Indian J Pathol Microbiol 2010;53:96100.

16. Sud A, Varma N, Marwaha RK, Patel FM, Trehan A, Singh S et al. Visceral leishmaniasis in a non-endemic area of India. Trop Doct. 2004;34:247-9.

17. Ahmad L, Yasin F, Iqbal K. Diagnosis of storage diseases in children by bone marrow examination. Pak Paed J 1995;19:15-7.

18. Ikram, N, Hassan K, Bukhari K. Spectrum of hematological lesions 
amongst children, as observed in 963 consecutive bone marrow biopsies. J Pak Inst Med Sci 2002;13:686-90.

19. Bain BJ. Bone marrow trephine biopsy. J Clin Pathol 2001;54:73742.
20. Chales K.S, Winfield DA, Angel C, Goepel J. Audit of bone marrow aspirates and trephine biopsies in multiple myeloma- a single center study. Clin lab hematol 2004;26:403-6.

21. Marsh JC, Ball SE, Cavenagh J et al. Guidelines for the diagnosis and management of aplastic anaemia. Br J Haematol. 2009;147:43-70. 